\title{
Cytokine profile and insulin antibody lgG subclasses in patients with recent onset Type 1 diabetes treated with oral insulin
}

\author{
L. Monetini ${ }^{1}$ M. G. Cavallo ${ }^{2}$ E. Sarugeri ${ }^{3}$ F. Sentinelli ${ }^{4}$ L. Stefanini ${ }^{1} \cdot$ E. Bosi $^{3} \cdot$ R. Thorpe Th. Pozzilli $^{1,6}$ • $^{5}$ \\ the Immunotherapy Diabetes (IMDIAB) group \\ ${ }^{1}$ Department of Endocrinology and Diabetes, University Campus Bio-Medico, Rome, Italy \\ ${ }^{2}$ Department of Clinical Medicine and Therapeutics, University "La Sapienza", Rome, Italy \\ 3 Internal Medicine, Diabetes and Endocrinology Unit, Vita-Salute San Raffaele University Hospital, Milan, Italy \\ ${ }^{4}$ Department of Clinical Sciences, Endocrinology, University "La Sapienza", Rome, Italy \\ ${ }^{5}$ Division of Immunobiology, National Institute for Biological Standards and Control, South Mimms, UK \\ ${ }^{6}$ Department of Diabetes, Institute of Cell and Molecular Science, Queen Mary College, St. Bartholomew's Hospital, London, UK
}

\section{Abstract}

Aims/hypothesis. Tolerance to orally administered antigens may be generated through the induction of $\mathrm{T}$ helper cell type 2 and $3(\mathrm{Th} 2 / \mathrm{Th} 3)$ regulatory cells. We previously reported that treatment of recent onset Type 1 diabetes with oral insulin had no effect on residual beta cell function. The aim of this study was to evaluate whether this treatment produces a deviation in the immune response, with polarisation of the cytokine pattern and the induction of a Th2-like antibody response. Methods. Mononuclear cells were collected from a total of 20 patients with Type 1 diabetes before and after 12 months of treatment with oral insulin $(n=11)$ or placebo $(n=9)$. Following stimulation of the cells with insulin or phytohaemagglutinin, levels of Th2 and Th3 cytokines (including TGF- $\beta$, IFN- $\gamma$, IL- 4 and IL-5) in the culture supernatants were assessed by ELISA. In addition, levels of total and specific insulin antibody IgG subclasses were measured by radioimmunoassay in serum samples drawn from 33 patients with Type 1 diabetes before and after 3, 6 and 12 months of therapy with oral insulin $(n=18)$ or placebo $(n=15)$.

Results. After 12 months of treatment, the release of TGF- $\beta$ was significantly higher in patients who re- ceived oral insulin compared with those who received placebo ( $p=0.025$ and $p=0.006$ for lymphocytes challenged with insulin and phytohaemagglutinin respectively). The two groups had similar levels of IL-4 and IL-5 both at baseline and after 12 months of treatment. The release of IFN- $\gamma$ was markedly reduced in patients treated with oral insulin compared with those who received placebo at the 12-month follow-up. Circulating levels of IgG1 and IgG3 subclasses directed against insulin were significantly lower in the oral insulin group than in the placebo group after 12 months of treatment $(p=0.05$ for IgG1 and $p=0.014$ for $\operatorname{IgG} 3)$.

Conclusions/interpretation. The increased TGF- $\beta$ release observed in patients treated with oral insulin suggests that a regulatory response can be induced in vivo by this treatment. The lower levels of insulin antibody IgG1 and IgG3 subclasses present in patients exposed to oral insulin are consistent with a Th2 deviation of the immune response. The failure of oral insulin treatment to provide any measurable clinical benefit may be due to the timing of treatment initiation.

Keywords Cytokines $\cdot$ IgG insulin antibodies · Oral insulin - Type 1 diabetes
Received: 2 December 2003 / Accepted: 12 July 2004

Published online: 22 October 2004

(C) Springer-Verlag 2004

P. Pozzilli ( )

Department of Endocrinology and Diabetes,

University Campus Bio-Medico, Via Longoni 83, 00155 Rome,

Italy

E-mail: p.pozzilli@unicampus.it

Tel.: +39-06-22541556, Fax: +39-06-22541336

\section{Introduction}

The induction of antigen-specific immune tolerance by the oral administration of soluble antigen has been widely investigated as a novel therapeutic approach in

Abbreviations: IA, insulin antibody $\cdot$ IMDIAB, immunotherapy diabetes - NOD, non-obese diabetic .

PHA, phytohaemagglutinin $\cdot$ PBMC, peripheral blood mononuclear cell $\cdot \mathrm{Th}$, T helper cell 
the treatment of autoimmune diseases [1]. The oral administration of low doses of auto-antigen modulates the immune system; however, the mechanism involved in this process has not been elucidated. One hypothesis is that oral administration of disease-associated antigen may act on gut immune function by up-regulating regulatory immune cells, which in turn suppresses the inflammatory response [2].

In Type 1 diabetes, beta cell destruction is thought to be mediated by a T helper cell type 1 (Th1) immune response in which pro-inflammatory Th1 cytokines (IL-1, IFN- $\gamma$ and TNF- $\alpha$ ) that are actively associated with 'destructive' insulitis are released by islet-infiltrating immunocompetent cells [3]. Animal models have been used to assess the ability of islet cell antigens administered via different routes (oral, intravenous, subcutaneous or nasal) to modulate the immune response and prevent or delay beta cell loss $[4,5,6,7,8]$. Animal models of Type 1 diabetes have demonstrated that the administration of diabetes-associated antigens, such as insulin or GAD, is effective in reducing or delaying the onset of disease $[8,9]$. These studies aimed to induce immune tolerance to beta cell antigens by promoting a deviation from a destructive Th1 response to a non-invasive/protective Th2/Th3 response. Regulatory Th3 cells secrete TGF- $\beta$, which is thought to be involved in the development of oral tolerance [10].

Immunoglobulin production is also regulated by cytokines. Although the underlying mechanisms associated with this are well understood in mice, they are still unclear in humans. In mice, IL-4 and TGF- $\beta$ induce the production of IgG1 and IgG2b antibodies respectively [11], whereas IFN- $\gamma$ stimulates the production of $\mathrm{IgG} 2 \mathrm{a}$ and $\mathrm{IgG} 3$ antibodies. In humans, IL-4 is thought to be responsible for switching to IgG4 and $\mathrm{IgE}$, whereas IFN- $\gamma$ probably induces the production of IgG1 and IgG3 antibodies. It is known that TGF- $\beta$ stimulates the production of $\operatorname{IgA}$ in both humans and mice $[12,13]$.

Based on these observations, two double-blind trials were designed to assess the effect of oral insulin administration on the induction of immune tolerance in patients with recent onset Type 1 diabetes $[14,15]$. In our own trial [14], as well as in the French trial of Chaillous et al. [15], the administration of oral insulin in addition to standard treatment with subcutaneous insulin failed to demonstrate a beneficial effect on residual beta cell function as assessed by $\mathrm{C}$-peptide secretion.

The aim of the present study was to assess whether the administration of oral insulin in addition to intensive insulin therapy promotes immunomodulation by inducing a Th2/Th3 polarisation of the cytokine pattern. Since immunoglobulin isotype switching is also controlled by cytokines [16], the effect of study treatment on the antibody response to insulin was also evaluated.

\section{Subjects and methods}

Subjects. Cytokine and/or insulin antibody measurements were studied in a total of 36 patients with Type 1 diabetes who were participating in the Immunotherapy Diabetes (IMDIAB) VII trial [14]. Venous blood samples were collected for assessment of cytokines from 20 of these patients at entry and after 12 months of treatment with either oral insulin $(n=9$, mean age $13.5 \pm 6.1$ years) or placebo ( $n=9$, mean age $16.2 \pm 10.4$ years) [11]. Insulin antibody assessments were performed in 33 of the 36 patients prior to randomisation and after 3, 6 and 12 months of treatment with oral insulin $(n=18$, mean age $12.7 \pm 6.6$ years) or placebo ( $n=15$, mean age $11.2 \pm 8.4$ years). Seventeen patients had both cytokine and insulin antibody levels assessed (nine treated with oral insulin and eight treated with placebo). The study was approved by the Ethical Committee of the University of Rome, La Sapienza, and was carried out in accordance with the Declaration of Helsinki. All patients gave their written informed consent to participate in the study.

Measurement of cytokines. Venous blood for cytokine assessment was drawn at diagnosis (before randomisation to either placebo or oral insulin) and after 12 months of treatment. Peripheral blood mononuclear cells (PBMCs) were isolated by FicollHypaque density gradient centrifugation at $1800 \mathrm{rev} / \mathrm{min}$ $(800 \mathrm{~g})$ for $30 \mathrm{~min}$ at room temperature. Cells were collected at the interface, washed twice and cultured at a concentration of $2 \times 10^{6} / \mathrm{ml}$ in 24 -well plates in the presence of phytohaemagglutinin (PHA, $10 \mu \mathrm{g} / \mathrm{ml})$, insulin $(200 \mathrm{IU} / \mathrm{ml})$ or medium alone for $48 \mathrm{~h}$ at $37{ }^{\circ} \mathrm{C}$ and $5 \% \mathrm{CO}_{2}$. Culture supernatants were then collected and frozen at $-20{ }^{\circ} \mathrm{C}$ until cytokine detection.

All cytokine measurements were performed by the same operator in a blinded manner. IL-4, IL-5, IFN- $\gamma$ and TGF- $\beta$ levels were measured by ELISA using commercially available antibodies. IL-5, IFN- $\gamma$ and IL-4 antibodies were obtained from Pharmingen (San Diego, Calif., USA) and DNAX (Palo Alto, Calif., USA), and TGF- $\beta$ antibodies were obtained from R\&D Systems (Minneapolis, Minn., USA). Briefly, plates were coated with antibodies $\left(100 \mu \mathrm{l}\right.$ per well) diluted in PBS overnight at $4{ }^{\circ} \mathrm{C}$ and washed twice with a solution of PBS containing $0.05 \%$ Tween 20 (PBS/Tween). Non-specific binding was blocked by the addition of a solution of PBS containing $0.05 \%$ BSA (PBS/BSA) for $30 \mathrm{~min}$. This and all subsequent steps were performed at room temperature. Appropriate dilutions of standards and samples were added in duplicate to the plates and left for 2-3 h. After washing with PBS/BSA the biotinylated detecting antibody (1:1000 dilution in PBS/BSA) was added and plates were incubated for $2 \mathrm{~h}$. After further washing with PBS/Tween, a streptavidin-horseradish peroxidase conjugated antibody (1:1000 dilution in PBS/BSA) was added and plates were incubated for $1 \mathrm{~h}$. After a final wash with PBS/Tween, 3,3',5,5'-tetramethylbenzidine substrate was added, and the reaction was stopped after $10-15 \mathrm{~min}$ by the addition of $50 \mu \mathrm{l}$ of $2 \mathrm{~mol} / \mathrm{l} \mathrm{H}_{2} \mathrm{SO}_{4}$ to each well. Plates were subsequently read at a wavelength of $450 \mathrm{~nm}$.

For TGF- $\beta$ detection, supernatants were activated prior to assay by the addition of an equal volume of $2.5 \mathrm{~mol} / \mathrm{l}$ acetic acid in $10 \mathrm{~mol} / \mathrm{l}$ urea at room temperature for $10 \mathrm{~min}$ and then neutralised to $\mathrm{pH} 7$ with $1 \mathrm{~mol} / \mathrm{l}$ Hepes in $2.7 \mathrm{~mol} / \mathrm{l} \mathrm{NaOH}$. Results are expressed as $\mathrm{ng} / \mathrm{ml}$ of culture supernatant.

Measurement of IgG subclasses. IgG subclasses of insulin antibodies (IA) were measured using the protein $\mathrm{A} / \mathrm{G}$ radiobinding assay, which was modified such that protein $\mathrm{A} / \mathrm{G}$ sepharose was substituted with IgG subclass-specific antibody-bound sepharose beads as previously described [17]. Insulin antibody concentrations were expressed as $\mathrm{nU} / \mathrm{ml}$ of serum as previously described [14]. 


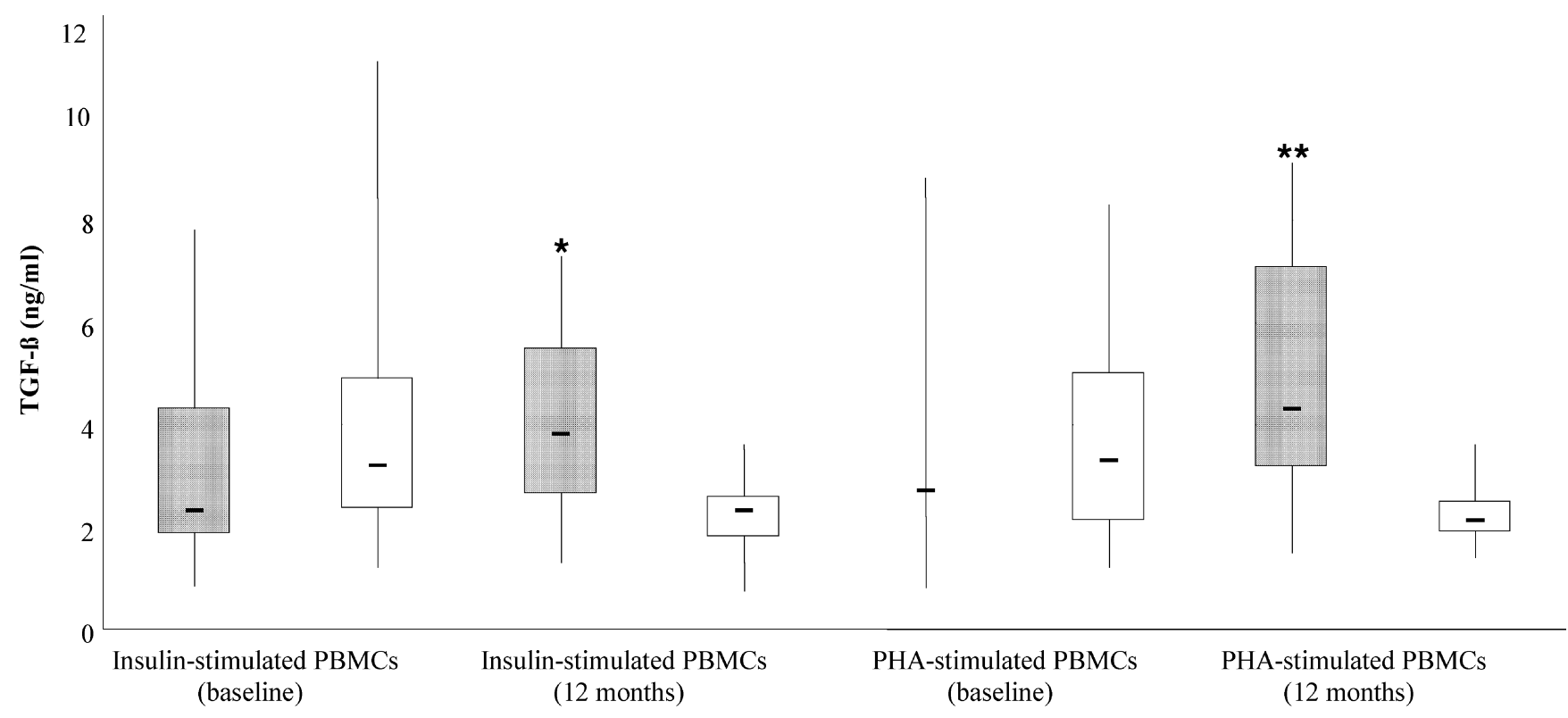

Fig. 1. TGF- $\beta$ production by insulin- or PHA-stimulated PBMCs from patients with Type 1 diabetes treated with oral insulin $(n=11$, grey boxes) or placebo $(n=9$, white boxes) at baseline

and after 12 months of treatment. Values are shown as box and whisker plots indicating the median, range and the $25 \mathrm{th} / 75 \mathrm{th}$ percentiles. $* p=0.025$ vs placebo; $* * p=0.006$ vs placebo

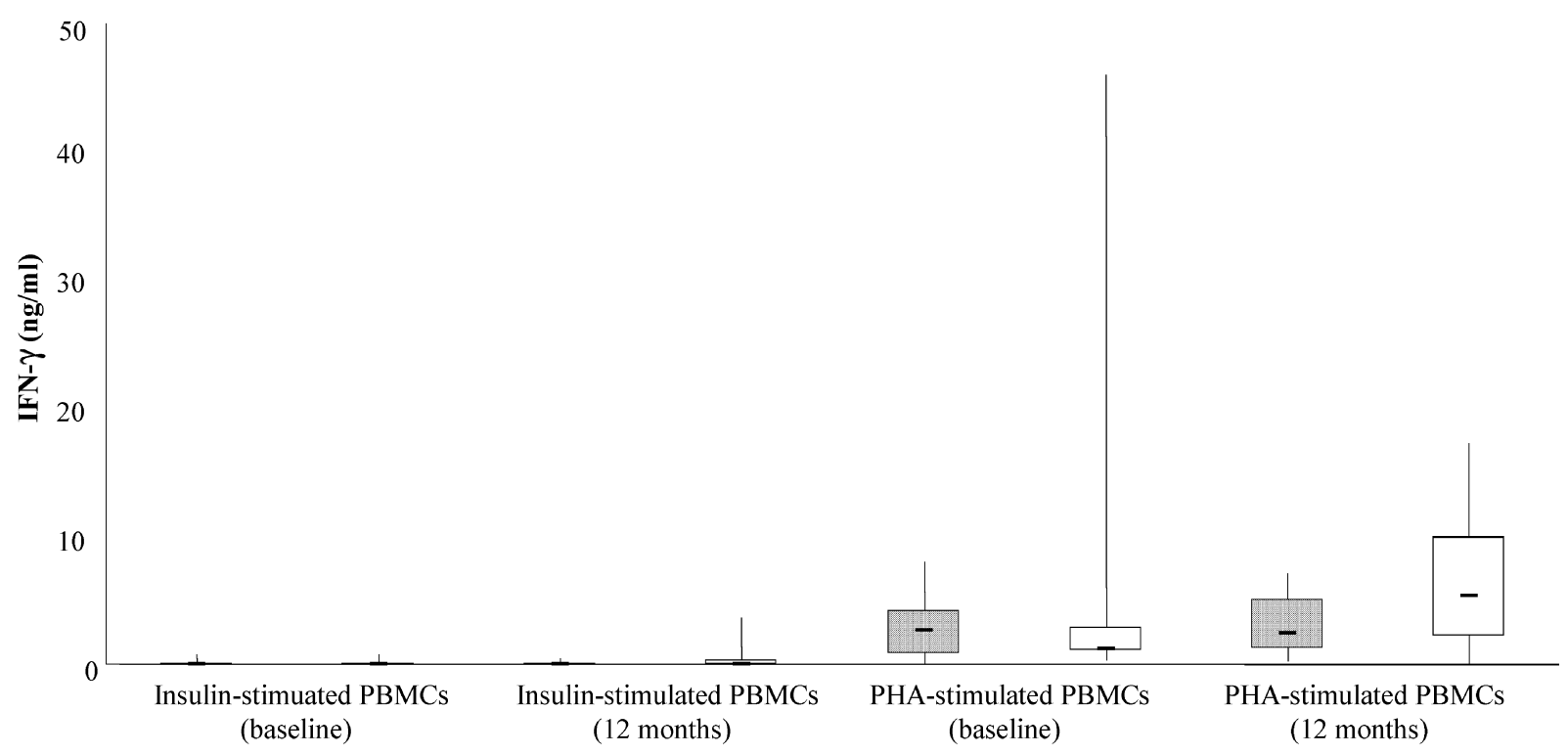

Fig. 2. IFN- $\gamma$ production by insulin- or PHA-stimulated PBMCs from patients with Type 1 diabetes treated with oral insulin ( $n=11$, grey boxes) or placebo $(n=9$, white boxes) at baseline and after 12 months of treatment. Values are shown as box and whisker plots indicating the median, range and the $25 \mathrm{th} / 75$ th percentiles. No significant differences were observed under the different experimental conditions

Statistical analysis. The Mann-Whitney U test for non-parametric values was used to compare median values for measurements of cytokines and $\mathrm{IgG}$ antibodies to insulin between the two groups. Results are shown as medians (ranges) unless stated otherwise. Any $p$ value less than 0.05 was considered to be statistically significant.

\section{Results}

Measurement of cytokines. Cytokine levels were measured in the culture supernatant of unstimulated (data not shown) and/or antigen-stimulated PBMCs from patients with Type 1 diabetes treated with oral insulin or placebo both at baseline and after 12 months of treatment. At diagnosis, the oral insulin and placebo groups had similar levels of cytokines (Figs. 1, 2, 3, 4).

TGF- $\beta$ levels were similar in the two groups at diagnosis $(3.50 \mathrm{ng} / \mathrm{ml}$ [1-9.5] for oral insulin patients vs $3.31 \mathrm{ng} / \mathrm{ml}$ [1.1-6.2] for placebo). After 12 months of treatment, spontaneous TGF- $\beta$ release was higher 


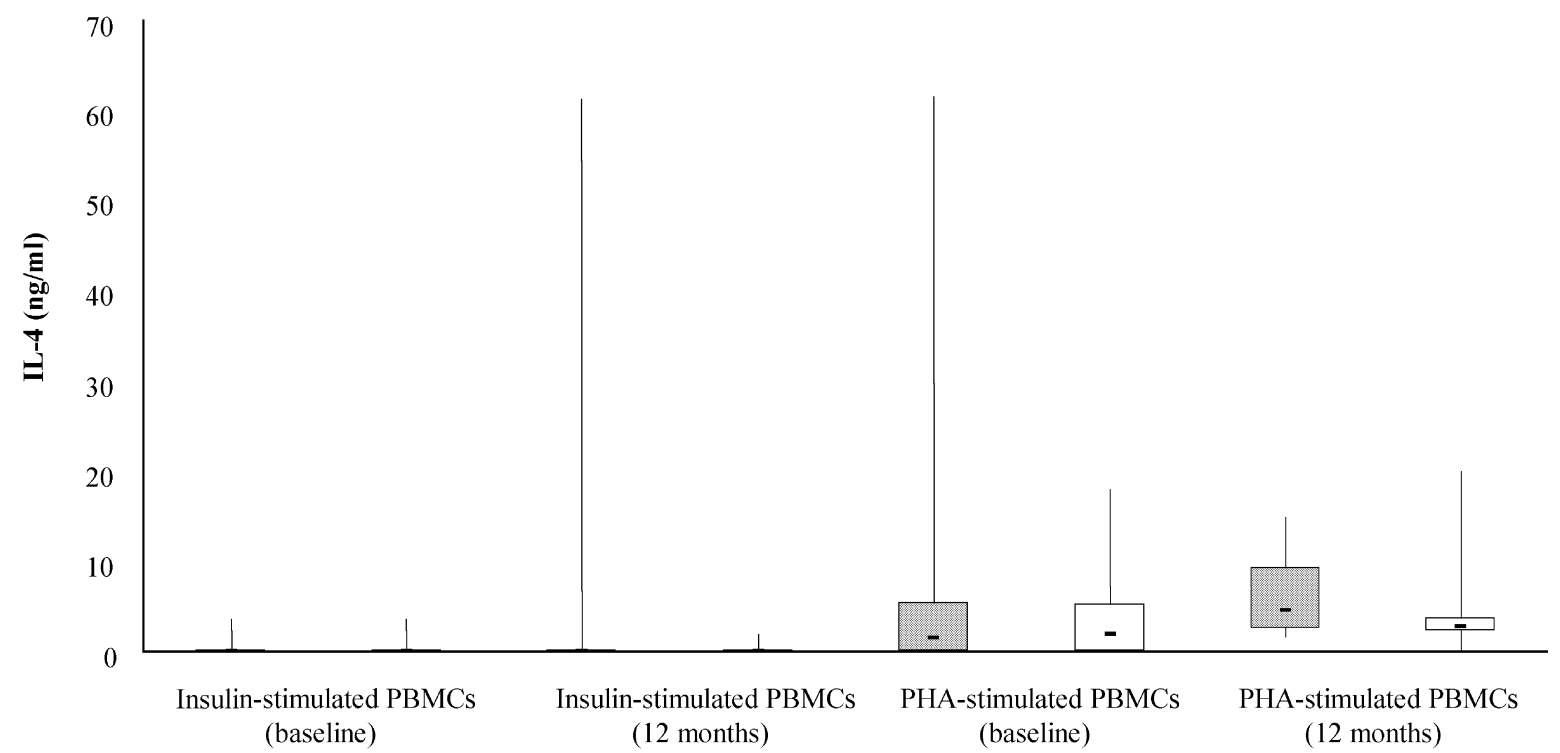

Fig. 3. IL-4 production by insulin- or PHA-stimulated PBMCs from treated with oral insulin $(n=11$, grey boxes) or placebo ( $n=9$, white boxes) at baseline and after 12 months of treatment. Values are shown as box and whisker plots indicating the

median, range and the 25 th/ 75 th percentiles. No significant differences were observed under the different experimental conditions

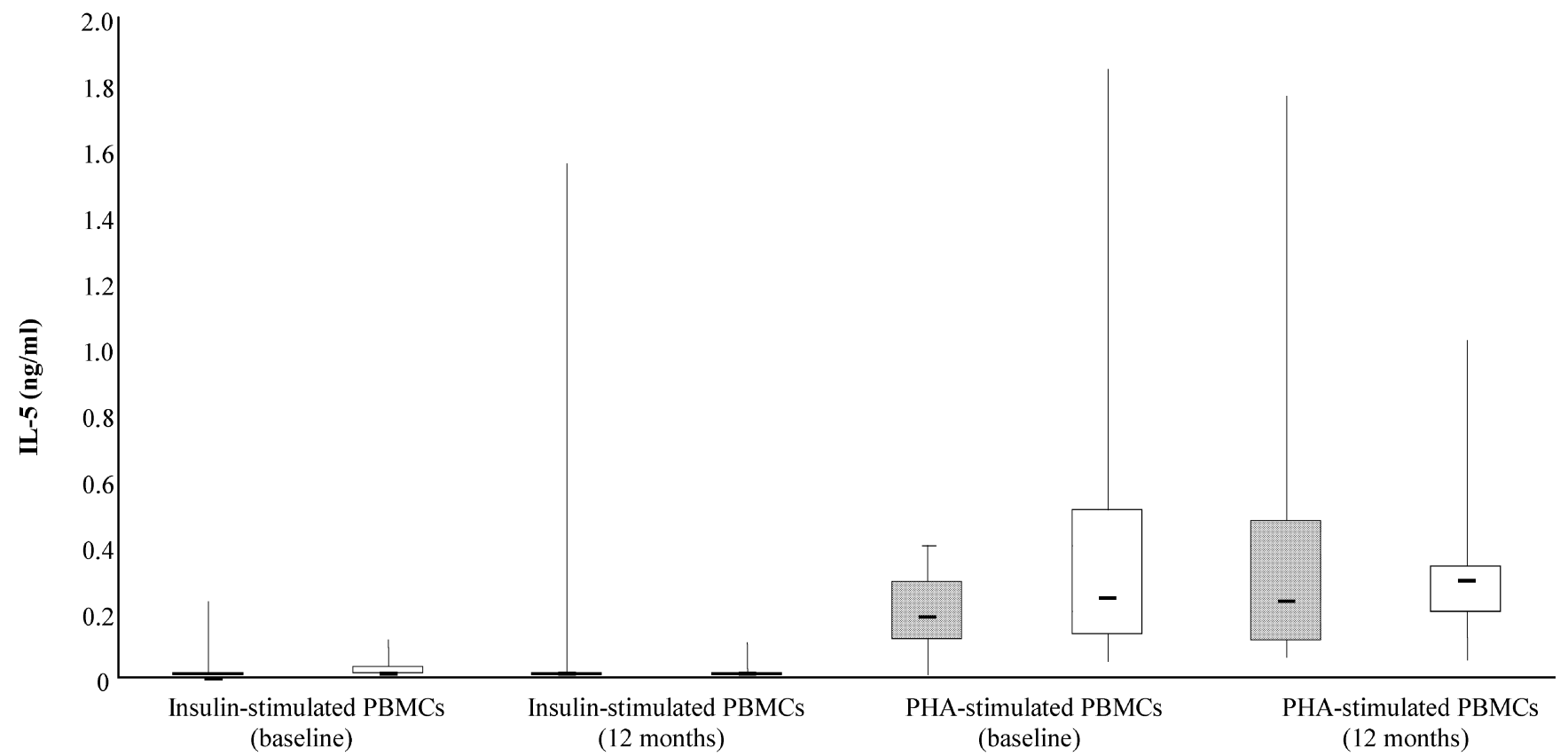

Fig. 4. IL-5 production by insulin- or PHA-stimulated PBMCs from patients with Type 1 diabetes treated with oral insulin ( $n=11$, grey boxes) or placebo $(n=9$, white boxes) at baseline and after 12 months of treatment. Values are shown as box and

whisker plots indicating the median, range and the $25 \mathrm{th} / 75$ th percentiles. No significant differences were observed under the different experimental conditions

in the oral insulin group than in the placebo group $(4.5 \mathrm{ng} / \mathrm{ml}[1.7-8]$ vs $1.90 \mathrm{ng} / \mathrm{ml}[1.1-6.2] ; p=0.05)$. At this time, a significant increase in TGF- $\beta$ was also detected in the PBMC culture supernatants of the oral insulin group compared with the placebo group following insulin or PHA stimulation $(p=0.025$ and

Fig. 5. Levels of IgG1-IA (a), IgG2-IA (b), IgG3-IA (c) and IgG4-IA (d) subclasses in patients treated with oral insulin ( $n=18$, grey boxes) or receiving placebo ( $n=15$, white boxes) at entry and after 3, 6 and 12 months of treatment. Values are shown as box and whisker plots indicating the median, range and the 25 th $/ 75$ th percentiles. $* p=0.05$ vs placebo; $* * p=0.014$ vs placebo 


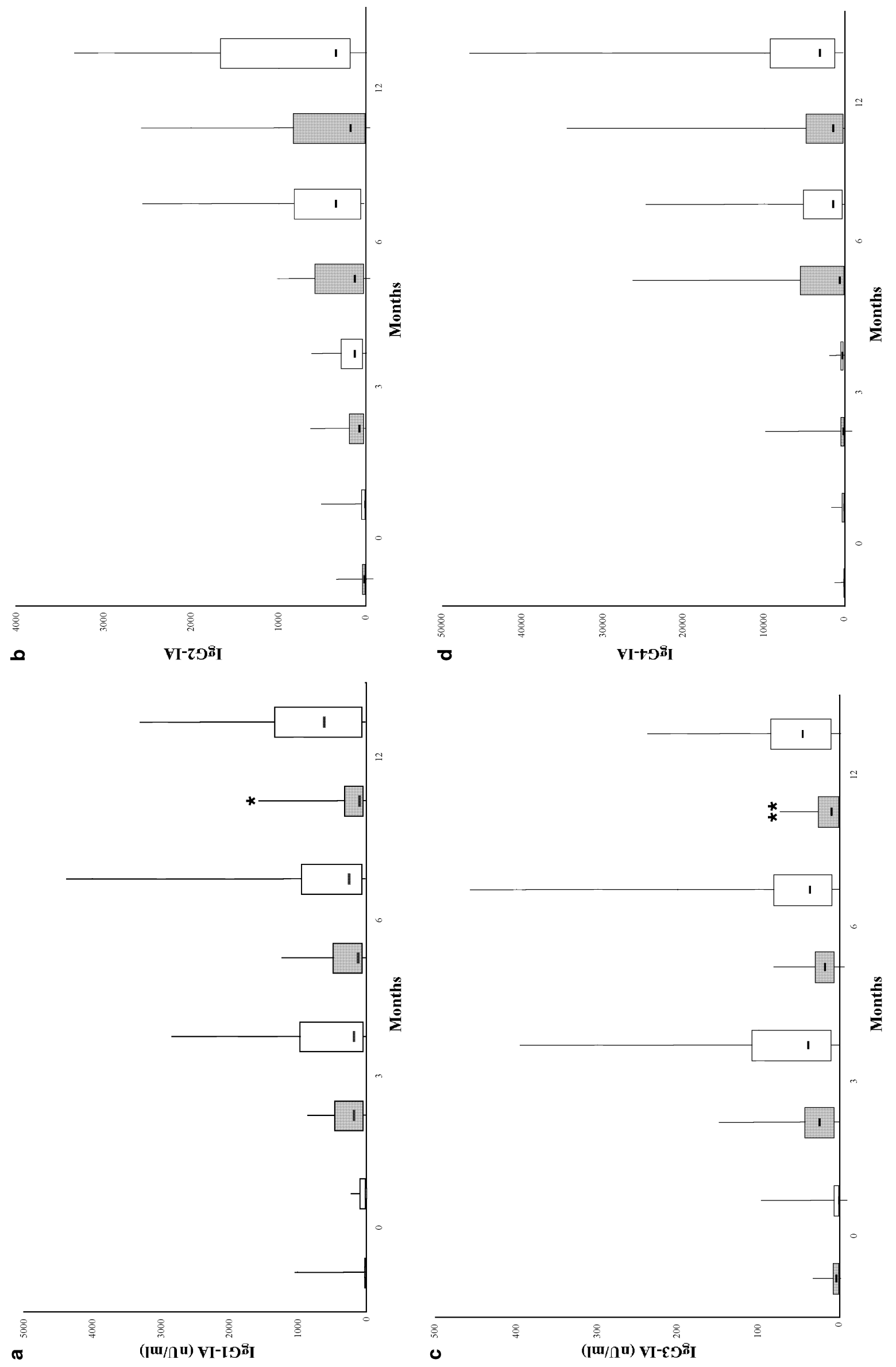


$p=0.006$ for lymphocytes challenged with insulin and PHA respectively) (Fig. 1).

No differences were observed with respect to IFN- $\gamma$ levels between the oral insulin and placebo groups either at entry $(0.03 \mathrm{ng} / \mathrm{ml}[0.006-0.246]$ vs $0.04 \mathrm{ng} / \mathrm{ml}$ [0.006-0.55] respectively) or at the 12 -month followup $(0.02 \mathrm{ng} / \mathrm{ml}$ [0.006-1.1] vs $0.04 \mathrm{ng} / \mathrm{ml}$ [0.01-0.246] respectively). Interestingly, PHA-stimulated IFN- $\gamma$ release was reduced in patients receiving oral insulin compared with those receiving placebo after 12 months of treatment. This difference was not statistically significant (Fig. 2); however, the variation in IFN- $\gamma$ levels between 0 and 12 months was significantly higher in patients receiving placebo $(p<0.05$, data not shown). There were no differences in insulin-stimulated IFN- $\gamma$ production between the groups either at baseline or after 12 months of treatment (Fig. 2).

Levels of insulin- or PHA-stimulated IL-4 and IL-5 release were similar in the two groups at both time points tested (Figs. 3, 4).

No differences were observed in culture supernatant of PBMC not stimulated with antigen between the groups (data not shown).

Measurement of IgG subclasses. Prior to the initiation of treatment with either oral insulin or placebo, no statistically significant differences were observed between the two randomised groups with respect to insulin antibody levels. At inclusion, 54.5\% (18 of 33) of patients tested positive for IA; IgG1 was observed to be the prevalent isotype at this time (data not shown). At 12 months, the frequency of IA-positive patients had increased up to $93.9 \%$ (31 of 33). Levels of the different IA IgG subclasses were not significantly different between the two groups at inclusion or after 3 or 6 months of treatment. However, serum levels of IgG1-IA and IgG3-IA were significantly lower in the oral insulin group than in the placebo group at the 12-month follow-up ( $p=0.05$ and $p=0.014$ for IgG1-IA and IgG3-IA respectively) (Fig. 5). No statistically significant differences in IgG2-IA and IgG4-IA levels were detected between the two groups during follow-up (Fig. 5).

No correlations were observed between TGF- $\beta$ and any of the IgG-IA subclasses; however, oral-insulintreated patients with higher TGF- $\beta$ levels had lower serum levels of IgG1-IA and IgG3-IA than placebotreated patients at 12 months (data not shown).

\section{Discussion}

This is the first study to investigate whether oral administration of insulin can modulate antigen-specific cellular and humoral immune responses in patients with recent onset Type 1 diabetes. The data presented show that after 1 year of treatment with oral insulin, relevant immunological changes are observed in terms of a Th2/Th3 polarisation of the cytokine pattern and a Th2-like antibody response. However, previously reported metabolic data from the IMDIAB VII trial showed that this treatment has no effect on residual beta cell function, since C-peptide levels, insulin requirements and glycated haemoglobin levels were comparable during follow-up in patients receiving oral insulin or placebo in addition to intensive insulin therapy [14]. A different study also found no differences between treatment groups in terms of C-peptide levels and antibody responses to different antigens, including insulin, islet cell cytoplasmic antigen, GAD and islet antigen 2 [15].

Oral treatment with auto-antigens such as GAD65, either alone or in combination with insulin, produced encouraging results in terms of delaying and/or blocking the development of diabetes in animal models [4]. Exposure to antigens by oral administration seems to act on gut immune function by activating local regulatory $\mathrm{T}$ cells to release specific cytokines with immunosuppressive functions [6]. One of these cytokines is TGF- $\beta$, which has previously been implicated in the induction of oral tolerance [10]. Several studies have discussed the potential of TGF- $\beta$ in the prevention of a number of autoimmune diseases, including rheumatoid arthritis, experimental allergic encephalomyelitis and Type 1 diabetes [18]. TGF- $\beta$ is secreted by Th3 lymphocytes and is known to exert its immunosuppressive role by regulating the proliferative responses of B lymphocytes, Th1 lymphocytes and cytotoxic T cells. It has been shown that TGF- $\beta$ suppresses IL-2dependent $\mathrm{T}$ cell proliferation by interfering with signal transduction [19]. Furthermore, TGF- $\beta$ controls immunoglobulin secretion and isotype switching [20]. Taken together, these findings suggest that TGF- $\beta$ plays a key role in the regulation of the immuno-inflammatory response.

In the present study, the up-regulation of TGF- $\beta$ production and concomitant down-regulation of IFN- $\gamma$ secretion in PHA-stimulated lymphocytes from patients treated with oral insulin suggests the occurrence of a shift from a potentially destructive Th1 response to a 'protective' Th2/Th3 reaction. In addition, a Th2 antibody response is indicated by the observed decrease in IgG3-IA levels after 12 months of treatment with oral insulin. Moreover, levels of IgG1-IA were lower in the oral insulin group than in the placebo group. This observation is in agreement with a previous study which showed that oral insulin administration is associated with lower levels of IgG1-IA after 3 months of treatment [15]. Conversely, subcutaneous insulin administration has been shown to induce high levels of IgG1-IA and a Th2-associated IgG4 response against insulin after 12 months of treatment [21].

Although our results are consistent with a deviation from a Th1-like to a Th2-like immune response, this did not delay disease progression in the patients treated with oral insulin. In other words, the shift in immune response was not associated with a clinical ben- 
efit in this case. One possibility is that the timing of treatment implementation-after disease onset-was too late to have a demonstrable effect on residual beta cell function [22]. However, in a recent trial performed on a large population of first- and second-degree relatives of Type 1 patients with diabetes, treatment with subcutaneous insulin also failed to either delay or block diabetes onset [23]. Data from animal models and trials in humans indicate that even if a shift from a Th1 to a Th2 immune response does occur, the development of resistance to Type 1 diabetes is not clearly associated with these immunological events in terms of a cause-effect relationship [24, 25, $26,27]$. In studies on non-obese diabetic (NOD) mice genetically deficient in IFN- $\gamma$, IL- 4 or IL- 10 , the rate of spontaneous development of diabetes was not altered by the absence of these cytokines [26]. Furthermore, the results of additional experiments in the same study suggested that protection from disease conferred by complete Freund's adjuvant or bacillus Calmette-Guerin vaccine was independent from the Th1/Th2 balance. In another study, transgenic mice overexpressing TGF- $\beta_{1}$ and TNF- $\alpha$ in pancreatic beta cells developed diabetes by the age of 4 months [27]. Thus, the clinical benefits of the immune modulation afforded by the oral administration of auto-antigens have yet to be determined.

Using animal models, many studies have indicated that cytokines play a key role in the development and progression of autoimmune diseases, including Type 1 diabetes, and may therefore prove useful in delaying and/or monitoring the disease process. It has recently been reported that progression to Type 1 diabetes correlates with an increase in Th1-like soluble cytokines in the peripheral blood of NOD mice [28].

In conclusion, our data suggest that a regulatory immune response can be generated by the oral administration of insulin in recent onset Type 1 diabetes; however, this effect has no clinically relevant beneficial impact on disease progression.

Acknowledgements. L. Monetini and M.G. Cavallo collaborated equally on this paper. This work was supported by grants from the Italian Ministry of Education, Universities and Research (MIUR, 40\%), the Italian Ministry of Health and the International Centre for the Study of Diabetes (CISD), Italy. The editorial assistance of T. Szendeffy is greatly appreciated.

\section{References}

1. Weiner H (1997) Oral tolerance: immune mechanisms and treatment of autoimmune diseases. Immunol Today $18: 335-343$

2. Alpan O (2001) Oral tolerance and gut-oriented immune response to dietary proteins. Curr Allergy Asthma Rep 1:572-577

3. Suarez-Pinzon WL, Rabinovitch A (2001) Approaches to Type 1 diabetes prevention by intervention in cytokine immunoregulatory circuits. Int J Exp Diabetes Res 2:3-17
4. Ramiya VK, Shang XZ, Wassefall CH, Maclaren NK (1997) Effect of oral and intravenous insulin and glutamic acid decarboxylase in NOD mice. Autoimmunity 26:139-151

5. Polanski M, Melican NS, Zhang J, Weiner HL (1997) Oral administration of the immunodominant B-chain of insulin reduces diabetes in a co-transfer model of diabetes in the NOD mouse and is associated with a switch from Th1 to Th2 cytokines. J Autoimmun 10:339-346

6. Maron R, Melican NS, Weiner HL (1999) Regulatory Th2type $\mathrm{T}$ cell lines against insulin and GAD peptides derived from orally- and nasally-treated NOD mice suppress diabetes. J Autoimmun 12:251-258

7. Maron R, Guerau-de-Arellano M, Zhang X, Weiner HL (2001) Oral administration of insulin to neonates suppresses spontaneous and cyclophosphamide induced diabetes in the NOD mouse. J Autoimmun 16:21-28

8. Aspord C, Thivolet C (2002) Nasal administration of CTBinsulin induces active tolerance against autoimmune diabetes in non-obese diabetic (NOD) mice. Clin Exp Immunol 130:204-211

9. Jun HS, Chung YH, Han J et al. (2002) Prevention of autoimmune diabetes by immunogene therapy using recombinant vaccinia virus expressing glutamic acid decarboxylase. Diabetologia 45:668-676

10. Suarez-Pinzon WL, Rabinovitich A (2001) Induction and mechanism of action of transforming growth factor-betasecreting Th3 regulatory cells. Immunol Rev 182:207-214

11. Abbas AK, Murphy KM, Sher A (1996) Functional diversity of helper T lymphocytes. Nature 383:787-793

12. Van Vlasselaer P, Punnonen J, De Vries JE (1992) Transforming growth factor- $\beta$ directs IgA switching in human B cells. J Immunol 148:2062-2067

13. Coffman RL, Lebman DA, Shrader B (1989) Transforming growth factor beta specifically enhances $\operatorname{IgA}$ production by lipopolysaccharide-stimulated murine B lymphocytes. J Exp Med 170:1039-1044

14. Pozzilli P, Pitocco D, Visalli N et al. (2000) No effect of oral insulin on residual beta-cell function in recent-onset Type 1 diabetes (the IMDIAB VII). Diabetologia 43:10001004

15. Chaillous L, Lefevre H, Thivolet $\mathrm{C}$ et al. (2000) Oral insulin administration and residual beta-cell function in recentonset Type 1 diabetes: a multicentre randomised controlled trial. Lancet 356:545-549

16. Quong MW, Harris DP, Swain SL, Murre C (1999) E2A activity is induced during $\mathrm{B}$-cell activation to promote immunoglobulin class switch recombination. EMBO J 18:6307-6318

17. Williams AJ, Bingley PJ, Chance RE, Gale EA (1999) Insulin autoantibodies: more specific than proinsulin autoantibodies for prediction of Type 1 diabetes. J Autoimmun 13:357-363

18. Weiner HL (2001) Induction and mechanism of action of transforming growth factor-beta-secreting Th3 regulatory cells. Immunol Rev 182:207-214

19. Han HS, Jun HS, Utsugi T, Yoon JW (1997) Molecular role of TGF- $\beta$, secreted from a new Type of CD4+ suppressor T cell, NY4.2, in the prevention of autoimmune IDDM in NOD mice. J Autoimmun 10:299-307

20. Snapper CM, Mond JJ (1993) Towards a comprehensive view of immunoglobulin class switching. Immunol Today 14:15-17

21. Fuchtenbusch M, Kredel K, Bonifacio E, Schnell O, Ziegler AG (2000) Exposure to exogenous insulin promotes IgG1 and the T-helper2-associated IgG4 responses to insulin but not to other islet autoantigens. Diabetes 49:918925 
22. Pozzilli P, Cavallo MG (2000) Oral insulin and the induction of tolerance in man; reality or fantasy? Diabetes Metab Res Rev 16:306-307

23. Diabetes Prevention Trial-Type 1 Diabetes Study Group (2002) Effects of insulin in relatives of patients with Type 1 diabetes mellitus. N Engl J Med 346:1685-1691

24. Hanninen A (2000) Prevention of autoimmune Type 1 diabetes via mucosal tolerance: is mucosal autoantigen administration as safe and effective as it should be? Scand J Immunol 52:217-225

25. Pozzilli P (2002) The DPT-1 trial: a negative result with lessons for future Type 1 diabetes prevention. Diabetes Metab Res Rev 18:257-259
26. Serreze DV, Chapman HD, Post CM, Johnson EA, SuarezPinzon WL, Rabinovich A (2001) Th1 to Th2 cytokine shifts in nonobese diabetic mice: sometimes an outcome, rather than the cause, of diabetes resistance elicited by immunostimulation. J Immunol 166:1352-1359

27. Sanvito F, Nicholas A, Herrera PL et al. (1995) TGF- $\beta 1$ overexpression in murine pancreas induces chronic pancreatitis and, together with TNF-alpha, triggers insulin-dependent diabetes. Biochem Biophys Res Commun 217:12791286

28. Schloot NC, Hanifi-Moghaddam P, Goebel C et al. (2002) Serum IFN-gamma and IL10 levels are associated with disease progression in non-obese mice. Diabetes Metab Res Rev 18:64-70 